This paper is a postprint of a paper submitted to and accepted for publication in IET Intelligent Transport Systems and is subject to Institution of Engineering and Technology Copyright. The copy of record is available at IET Digital Library.

Official URL: http://dx.doi.org/10.1049/iet-its:20070060

The full citation for this paper is:

Hounsell, N.B., Shrestha, B.P., Head, J.R., Palmer, S. and Bowen, T. (2008) The way ahead for London's bus priority at traffic signals. IET Intelligent Transport Systems, 2, (3), 193-200. (doi:10.1049/iet-its:20070060) 


\title{
The Way Ahead for London's Bus Priority at Traffic Signals
}

\author{
N. B. Hounsell*, B. P. Shrestha*, J. R. Head ${ }^{+}$, S. Palmer^^${ }^{\wedge}$ and T. Bowen^ \\ *Transportation Research Group, University of Southampton \\ Southampton, United Kingdom \\ Email: N.B.Hounsell@ soton.ac.uk \\ Tel: +44 (0) 2380593705; Fax +44(0)2380593152 \\ ${ }^{+}$Consultant to Bus Priority Team, Transport for London \\ London, United Kingdom \\ ${ }^{\wedge}$ Bus Priority Team, Surface Transport, Transport for London \\ London, United Kingdom
}

\section{Background}

London has a long history of success in bus priority in all its forms, as one means of supporting efficient public transport operations. This has been possible because of the regulated nature of the bus operations in London (unlike the rest of the UK), which has allowed long-term planning and sustained investment. Implementation of bus priority at traffic signals has grown steadily since the large-scale trial conducted in the SELKENT (South East London and KENT) area of London in 1987. With the development of technology in the area of detection techniques, traffic signal systems and communication systems, London has updated its bus priority system to keep up with this pace of change. For example, the bus transponder/loop-antenna technology used for Selective Vehicle Detection (SVD) has been gradually replaced in recent years with the use of roadside beacons, using technology derived from the COUNTDOWN system for passenger information at bus stops. The beacon system [1] solved some of the drawbacks of loop detectors (e.g. vulnerability of the loop to damage), but is still a rigid system with costly installation, maintenance and repositioning (if needed).

Recently, Transport for London (TfL) has procured a modern Automatic Vehicle Location (AVL) system for fleet management, passenger information and bus priority. The new system is known as iBUS [2] and is based on Global Positioning System (GPS) and supporting technologies for bus location. Since this system eliminates the need for on-street hardware for detecting buses and requesting priority, it provides more flexibility and opportunity for using bus detectors. That makes it now feasible to use more than one detector per approach, if required, taking advantage of the 'virtual' detection concept. In this concept, the detection points are stored in the on-board computer without their physical presence in the field and hence are known as 'virtual' detectors. This provides an opportunity to enhance bus priority considerably through improved methods of control giving potentially greater benefits. For example, an exit detector could be used to curtail green after a bus crosses the stopline. Despite being flexible and versatile, bus location based on GPS is inevitably less accurate than location based on fixed infrastructure (inductive loops or beacons). Locational error could result for example, in some buses being incorrectly located further away from the junction than they actually are, so that they may miss the given priority action, particularly green extensions. 
This paper first summarises how bus priority at traffic signals works within iBUS, and then explores the effect of challenges posed by GPS locational error followed by a discussion of opportunities provided. The paper is based on various studies carried out by Transportation Research Group (TRG) at the University of Southampton for Transport for London.

\section{GPS based detection for bus priority: London's iBUS system}

Bus priority at traffic signals can be implemented using various priority architectures. With a growing number of differential priority schemes, it is apparent that a wide range of architectures are being employed in different cities across the globe [3]. A comparison of the effectiveness of these different bus priority architectures on the basis of their important aspects and options available was carried out in earlier research [4]. In its simplest form of giving priority using GPS, a bus is detected on the approach of a traffic signal using its GPS location and the priority is activated. This simple representation of GPS use in bus priority at traffic signals controlled by SCOOT UTC is shown in Figure 1.

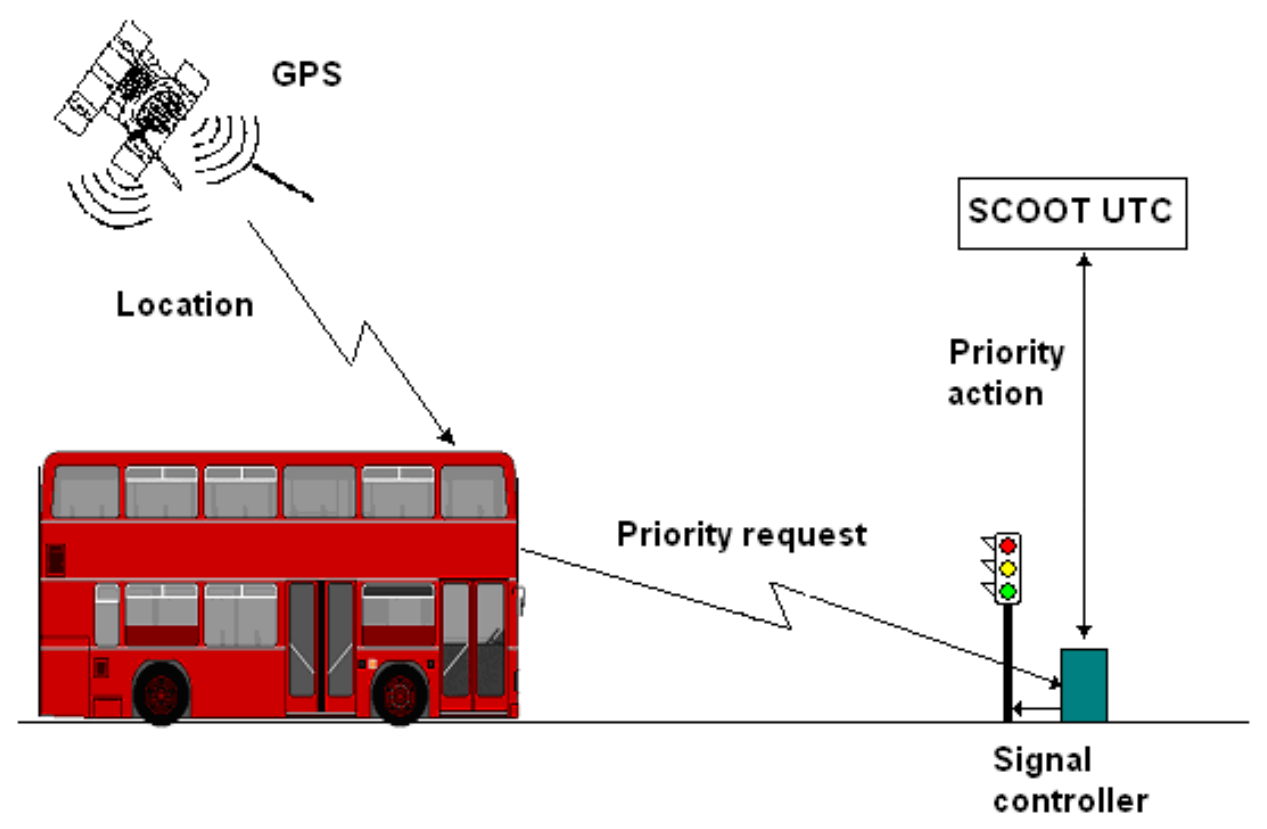

Figure 1: A simple representation of bus priority at traffic signals using GPS

With this priority architecture currently directly replacing beacon-based detection in London, the role of GPS is to detect buses at a predefined location on the approach to a traffic signal. Each bus regularly gets its location (e.g. every second) from its GPS receiver onboard. In places where GPS accuracy is particularly important (e.g. at bus stops), GPS location information may be aided with additional equipment such as its door closing sensor [5]. As a bus arrives at a traffic signal, the bus is detected by comparing its location with the predefined location of the detection point(s) on the route. The detection points are stored in the on-board computer and are also known as virtual detectors (as they have no physical presence). Once a bus is detected, a priority request is sent to the traffic signal controller. Within the constraint of the amount of priority time available, the priority request is implemented mainly in two ways: extensions and recalls. An extension is the method where the present green time is extended, if it is expected that the bus detected would otherwise just miss the present green period. A recall is the method where the green time is recalled more 
quickly if the bus is detected in the red period and is expected to arrive at the stopline before the start of the next green period. Facilities to compensate traffic on non-priority stages are also usually provided according to the type of signal control (e.g. SCOOT).

Even though a GPS based detection system provides flexibility by avoiding the need for physical detection infrastructure, its main drawback is the locational error associated with it. The various sources of the GPS locational error are given in earlier research [6]. As a result of the locational error, the actual distance from bus detection to the stopline may be different even though the detection is done at a predefined location (i.e. known distance from the stopline). If a bus is detected upstream of the predefined detector location as a result of GPS error, the journey time it needs may be more than the estimated time. Hence the bus may not clear the stopline within the present green period extended at the time of detection. This results in loss of bus priority benefits when compared to the 'no error' case. Although it is obvious that the extent of the estimation error and hence the loss of the priority benefits depends on the extent of the locational error, no research was available to quantify the extent of the effect. It is to be noted that GPS-based location systems are often aided by some additional technologies such as the vehicle's odometer and map matching to improve locational accuracy. The effect of such technology, however, depends on the site specific environment (e.g. map matching could be particularly valuable in more enclosed environments where GPS-based location is less reliable). This lack of information on the effect of the locational error posed a challenge in implementing GPS-based bus priority at traffic signals. This challenge is analysed in the next section both theoretically and then using results from simulation modelling to quantify the likely extent of the effect.

\section{Challenges}

The impact of GPS locational error on bus priority benefits is mainly due to the number of buses missing given priority extensions which is vulnerable to the variability in the bus journey time to the traffic signal. Before analysing the effect of GPS error on the bus delay savings, it is necessary to discuss the current practice in London for taking account of the existing journey time variability between the bus detector and the stopline. In field, the bus journey time between a detector and the stopline varies from one bus to another. This variation in the journey time is taken into account by adding an extra time on the top of the average journey time (bjyt) when estimating the time required for a bus to clear the downstream stopline. Here, 'bjyt' is a SCOOT parameter representing average free-flow bus journey time between the bus detection point and the traffic signal stopline. The extra time is known as the 'busvary' parameter (bvary) in SCOOT and is set in such a way that it makes sure that most of the buses get through within the estimated time. The higher the value of busvary, the more buses will get through on extensions (fewer buses will miss given extensions). However, a higher value of busvary results in more wasted time for some buses (those with higher than average speed) and this reduces efficiency of the traffic control. There is therefore a trade-off between the two considerations. Earlier research [7] gave relationships to calculate journey time variability for different detector distances and the corresponding busvary needed to ensure that most of the buses clear through in the present green time. Using these busvary values, a small proportion of buses may still miss the priority extensions given. However, with GPS locational error, the journey time variability increases and more buses miss priority extensions as a result. 


\subsection{Theoretical analysis}

The increase in journey time variability with GPS-based detection is based on: the existing bus journey time variability $\left(\sigma_{\mathrm{JT}}\right)$ and the variability due to the GPS error $\left(\sigma_{\mathrm{GPS}}\right)$. Since these two elements are additive and are assumed to be independent of one another, the resulting total standard deviation (SD) is given by:

$$
\mathrm{SD}=\sqrt{{\sigma_{\mathrm{JT}}}^{2}+{\sigma_{\mathrm{GPS}}}^{2}}
$$

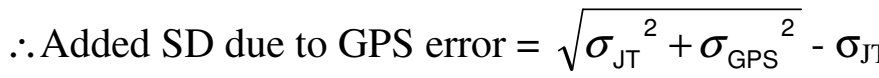

This relationship shows that the added variability (standard deviation) due to GPS error is greatly influenced by the bus journey time variability $\left(\sigma_{\mathrm{JT}}\right)$. If the bus journey time variability $\left(\sigma_{\mathrm{JT}}\right)$ is higher, the added variability due to GPS error is smaller and vice versa. Earlier research [7] showed that a longer detector distance results in higher bus journey time variability than a shorter detector distance. Hence the added variability due to GPS reduces with the increase in the detector distance. So, the impact of GPS locational error is proportionately smaller for longer detector distances.

This added bus journey time variability increases the percentages of buses missing awarded extensions (more than the existing percentage for a given value of busvary). Assuming the bus journey time variability follows a normal distribution profile, the percentage of buses having journey time variability more than busvary can be calculated using the standard normal distribution. For example, if the standard deviation is 2 seconds and the busvary used is 3 seconds, then the theoretical percentage of buses missing extensions would be $6.68 \%$ (derived from the value 0.9332 obtained for a $\mathrm{z}$ value of 1.5 ). The increase in the percentage of buses missing extensions (in comparison to that for existing variability) is due to the increased variability as a result of GPS error. This percentage is related to the percentage of buses that are getting priority extensions and not the percentage of all buses. The percentage of buses getting priority extensions is variable and needs to be taken into account when assessing the overall effect of GPS error.

The opportunity for priority extensions depends on the time window during which a bus can trigger priority extensions. This depends on the journey time between the detector and the stopline. The longer the journey time the more is the opportunity for a bus to request priority extensions, if needed. Theoretically, the opportunity depends on the journey time between the detector and the stopline and any extra time added for variability. The theoretical percentage of buses getting priority extensions in a cycle can be calculated as:

Percentage of extension awards $=\frac{(\text { bjyt }+ \text { busvary })}{\text { cycle time }} * 100$

Equation (3) shows that the percentage of extension awards increases with the detector distances as the bus journey time (bjyt) increases. However, it is to be noted that there is a practical maximum value for extensions which limits the detector location and the bus journey time. 20 seconds is commonly used in London as the limiting value for extensions to avoid unacceptable delay to non-priority traffic. Since the impact of GPS locational error is 
mainly on priority extensions, the impact of GPS locational could be felt more at longer distances with more number of extensions.

Using this percentage of buses getting priority along with the percentage of buses missing extensions due to GPS locational error, the overall theoretical percentage of buses (out of total buses) missing extensions can be calculated. Various calculations made to evaluate these values are given in Table 1. The existing journey time variability (row 1) and busvary (row 5) used are calculated using the relationship from earlier research [7] for Ealing. The average bus speed used for calculating these values is $10 \mathrm{~m} / \mathrm{s}$. The standard deviation of the GPS locational error is assumed to be $10 \mathrm{~m}$ for the calculation purpose (row 2). The resulting standard deviation (row 3) as a result of the existing journey time standard deviation and the GPS locational error is calculated using relationship (a). The percentages of buses missing extensions for different variability and the corresponding busvary are shown in rows 6 and 7 . Row 8 shows the increase in the percentage of buses missing (difference of row 7 and row 6) due to the increased variability as a result of GPS error. The theoretical percentage of the buses getting extensions (row 9) is calculated assuming a cycle time of 80 seconds. The overall percentage of buses missing extensions due GPS locational error (row 10) is calculated by multiplying row 8 and row 9 .

Table 1: Effect of GPS locational error on buses missing priority extensions

\begin{tabular}{|c|l|r|r|r|r|r|}
\hline \multirow{2}{*}{ Row } & \multicolumn{5}{|c|}{ Particulars } & \multicolumn{5}{c|}{ Detector distance $(\mathrm{m})$} \\
\cline { 3 - 7 } & & 50 & \multicolumn{1}{c|}{70} & 100 & 130 & 150 \\
\hline 1 & Existing journey time SD (no error) (s) & 0.83 & 1.16 & 1.66 & 2.16 & 2.49 \\
\hline 2 & Journey time for GPS error SD of 10m (s) & 1 & 1 & 1 & 1 & 1 \\
\hline 3 & Resulting journey time SD (with error) (s) & 1.3 & 1.53 & 1.94 & 2.38 & 2.68 \\
\hline 4 & Added SD due to GPS error (s) & $\mathbf{0 . 5 3}$ & $\mathbf{0 . 3 7}$ & $\mathbf{0 . 2 8}$ & $\mathbf{0 . 2 2}$ & $\mathbf{0 . 1 9}$ \\
\hline 5 & Busvary for existing journey time (s) & 2 & 2 & 3 & 3 & 4 \\
\hline 6 & $\begin{array}{l}\text { Percentage of buses missing extensions with no } \\
\text { locational error (\%) }\end{array}$ & 0.8 & 4.3 & 3.5 & 8.2 & 5.4 \\
\hline 7 & $\begin{array}{l}\text { Percentage of buses missing extensions with } \\
\text { GPS error (\%) }\end{array}$ & 6.2 & 9.6 & 6.1 & 10.4 & 6.8 \\
\hline 8 & $\begin{array}{l}\text { Extra percentage of buses missing } \\
\text { extensions due to GPS error (\%) }\end{array}$ & $\mathbf{5 . 4 0}$ & $\mathbf{5 . 3 0}$ & $\mathbf{2 . 6 0}$ & $\mathbf{2 . 2 0}$ & $\mathbf{1 . 4 0}$ \\
\hline 9 & $\begin{array}{l}\text { Theoretical percentage of buses getting } \\
\text { extensions (\%) }\end{array}$ & $\mathbf{8 . 8}$ & $\mathbf{1 1 . 3}$ & $\mathbf{1 6 . 3}$ & $\mathbf{2 0 . 0}$ & $\mathbf{2 3 . 8}$ \\
\hline 10 & $\begin{array}{l}\text { Percentage of buses (out of total buses) } \\
\text { missing extensions due to GPS error }(\%)\end{array}$ & $\mathbf{0 . 4 7}$ & $\mathbf{0 . 6 0}$ & $\mathbf{0 . 4 2}$ & $\mathbf{0 . 4 4}$ & $\mathbf{0 . 3 3}$ \\
\hline
\end{tabular}

Table 1 shows that the added variability due to the GPS error is greater at shorter detector distances and, consequently, the extra percentage of buses missing an awarded extension due to GPS error is also greater. In contrast, the number of buses getting extensions increases as detector distance increases. As a result, the percentage of buses missing extensions due to GPS locational error is almost constant for all the detector distances (approximately 0.4\%). Even though the percentage is very small, its impact is noticeable as the buses missing extensions will face a delay equal to at least the whole red period for the stage. If the red period for a stage is 40 seconds, then the extra delay is around 0.2 seconds/bus. This analysis shows that the impact of GPS locational error is relatively small (depending on the existing variability) and is nearly constant for all the detector distances. To verify the results more 
robustly taking account of the variation in the bus arrival time and priority requests, a further study was carried out with using simulation modelling of a traffic signal.

\subsection{Simulation modelling}

The study of the impacts of GPS for bus location for bus priority purposes was further investigated using TRG's microscopic simulation model SIMBOL (SImulation Model for Bus priOrity at traffic signaLs). SIMBOL [8] has been designed specifically to model alternative bus priority strategies at traffic signals, including a simple representation of general traffic. The model provides options to investigate the impact of bus priority on general traffic and vice-versa in detail without the influence of the randomness associated with general traffic behaviour. This model has been used in TRG for various bus priority studies (e.g. [8], [9] and [10]) in the last few years.

This study was based on the modelling of a signalised junction with two conflicting approach roads: a main road and a side road, with buses on the main road only. The traffic signal modelled here was a fixed time signal with 2-stage signal operation, a cycle time of 80 seconds, inter-green periods of 10 seconds and effective green durations of 40 seconds and 20 seconds for the two stages. Priority was given to buses in the form of "extensions" and "recalls" for individually detected buses, based on the estimated arrival time of the bus at the traffic signal. These facilities, and the controlling parameters, were those used currently for bus priority in the SCOOT UTC system. The maximum length of extension allowed was set to 20 seconds. Typically the actual degree of saturation was $80 \%$ and a target saturation parameter of $110 \%$ was used for recalls, resulting in a maximum amount of time gained for the bus of 5 seconds through recalls. Cars were generated on both approaches to the junction at regular intervals with flow values of 1440 vehicles/hour on the main road and 720 vehicles/hour on the side road.

For the purposes of this study, GPS locations were assumed to be available $100 \%$ of the time. (This is not the case in reality, due to masking by tall buildings, etc. However, in such cases, supplementary systems such as the bus odometer will 'take-over' to provide the location estimate). GPS location errors were considered to be random, with an average error of zero and a maximum error of three times the standard deviation, as typical from recent evidence. Two values of standard deviation were considered in the analyses presented in this paper: $5 \mathrm{~m}$ and $10 \mathrm{~m}$. GPS positions were sampled every second, which is consistent with the GPS 'polling rates' reported in the literature (e.g. [11]).

Simulations were carried out for different levels of GPS errors for different detector positions. Figure 2 shows the change in bus delay savings as a result of GPS error for various detector distances.

The results (Figure 2) show that increasing GPS error tends to reduce bus delay savings but only by a relatively small amount $(\sim 2-5 \%)$ within the range of errors concerned. This is probably due to the fact that the existing bus journey time variability is greater than the variability due to the GPS error (as evident in Table 1). Since the bus journey time variability is already catered for by the use of the SCOOT busvary parameter, the additional levels of GPS error considered here make lesser impact. Furthermore, the effect of the GPS error appears to be more or less constant over all of the detector positions considered: from $10 \mathrm{~m}$ up to $150 \mathrm{~m}$. This can be explained by considering that GPS error only has a significant impact 
on extensions, so it will tend to have a greater impact when there are more extensions. More extensions tend to occur at greater detection distances where existing bus journey variability is higher so the added increase in the variability due to GPS error has a lesser effect. These two conflicting impacts balance each other to give a more or less constant effect. These simulation results are found to be consistent with the theoretical analysis carried out earlier.

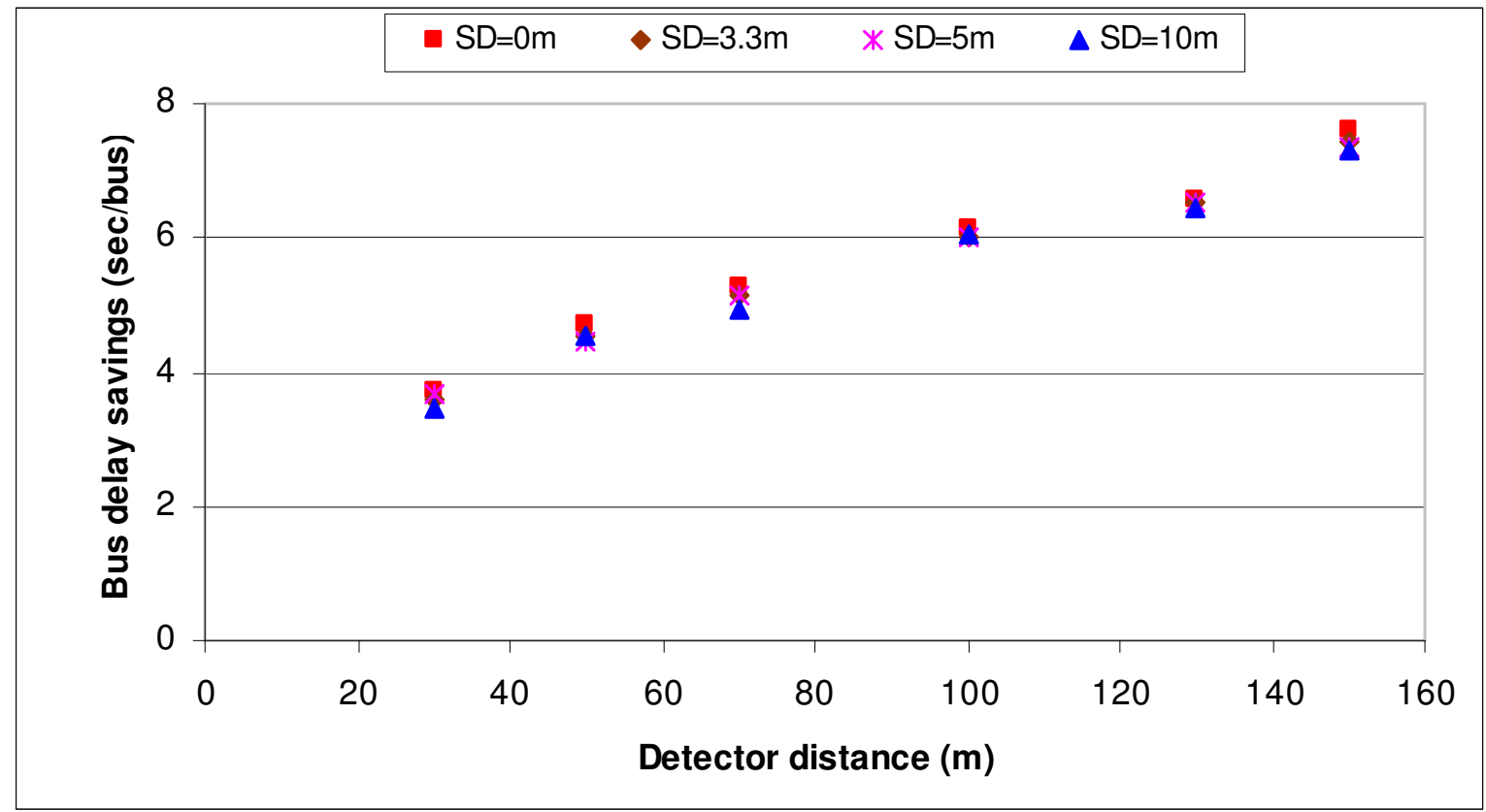

Figure 2: Effect of GPS locational error on bus delay savings

In addition to the bus delay savings, further analysis was also carried out to assess delays to the non-priority traffic (Figure 3). Delay savings for buses, main road traffic and side road traffic were analysed in terms of total person delay savings using an average occupancy of 13.2 persons per bus and 1.54 persons per car (COBA, 2002).

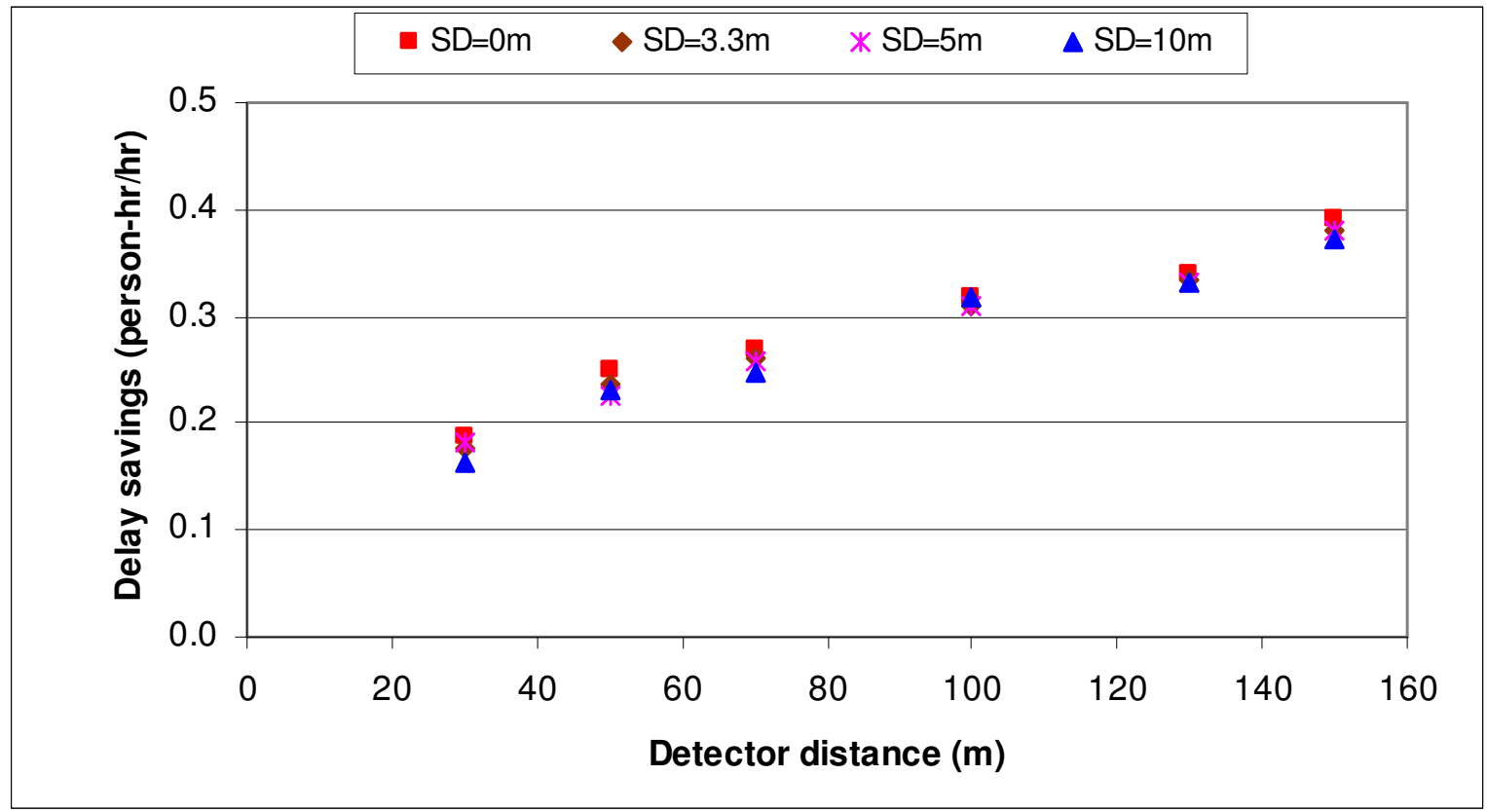

Figure 3: Effect of GPS locational error on total person delay savings 
Figure 3 shows that increasing GPS error tends to reduce total person delay savings but only by a very small amount. The trend here is similar to that for the bus delay savings shown in Figure 2. This is due to the fact that the impact of GPS error on general traffic was negligible as the reduction in bus delay savings is mainly due to the number of buses missing awarded extensions; and not due to the reduced number of extensions awarded. These results showed that the effects of typical GPS locational error on the benefits from bus priority are small. Recent research has shown that these should be more than offset by additional benefits due to the additional capabilities such as multiple detection. Some of the potential implementations that are possible using iBUS system are discussed in the next section.

\section{THE WAY AHEAD}

The flexibility of iBUS opens up many possibilities for improving bus priority at traffic signals. Three issues considered in this paper for the upgraded bus priority system are: (i) different bus detection methods; (ii) differential priority; and (iii) system monitoring.

\subsection{Bus detection methods}

The bus detection in London has always been downstream of bus stops due to the big variability in dwell time at bus stops. Research has shown that detecting buses upstream of a bus stop can be beneficial where the bus stop is close to the stopline and bus dwell time is fairly constant/predictable (This is happening increasingly with new multi-door buses and pre-paid ticketing in London). Such a method using a single detector on an approach could still be provided by current beacon detection. However, a combination of detectors (more than one detector) is costly to implement using beacons. The provision of more than one virtual detection point (without the need for physical infrastructure) in iBUS opens up the opportunity to implement a combination of detectors for different purposes. For example, an extra detector could be used (called a secondary detector here) downstream of the first detector (called a primary detector here) to review the priority triggered earlier (by the primary detector). This will help slower buses which may not otherwise reach the stopline before the end of present green time to obtain an additional extension. This is particularly the case when buses are detected upstream of a bus stop where dwell time can vary considerably, affecting the bus journey time to the stopline. Furthermore, an extra detector can be used as a cancel detector placed near the stopline to curtail (cancel) a priority action once the bus has passed through the signals. Timely cancellation of bus priority removes the safety margin currently in use to protect buses from journey time variations. The cancellation of such time reduces the extra delay to side road traffic and improves junction efficiency.

Research [9] showed that bus priority is beneficial when both of these detection options are implemented in addition to the normal detector. This arrangement is particularly beneficial where the bus stop is close to the traffic signals $(<50 \mathrm{~m})$, where the bus priority benefits from detection downstream of the stop are likely to be low. A typical layout of these various detectors is shown in Figure 4. Such layout of detectors would have been costly with rigid detection systems such as loop detectors or beacons. However, in iBUS, the layout is possible to implement as these detectors are just programmed locations inside the bus computer. 


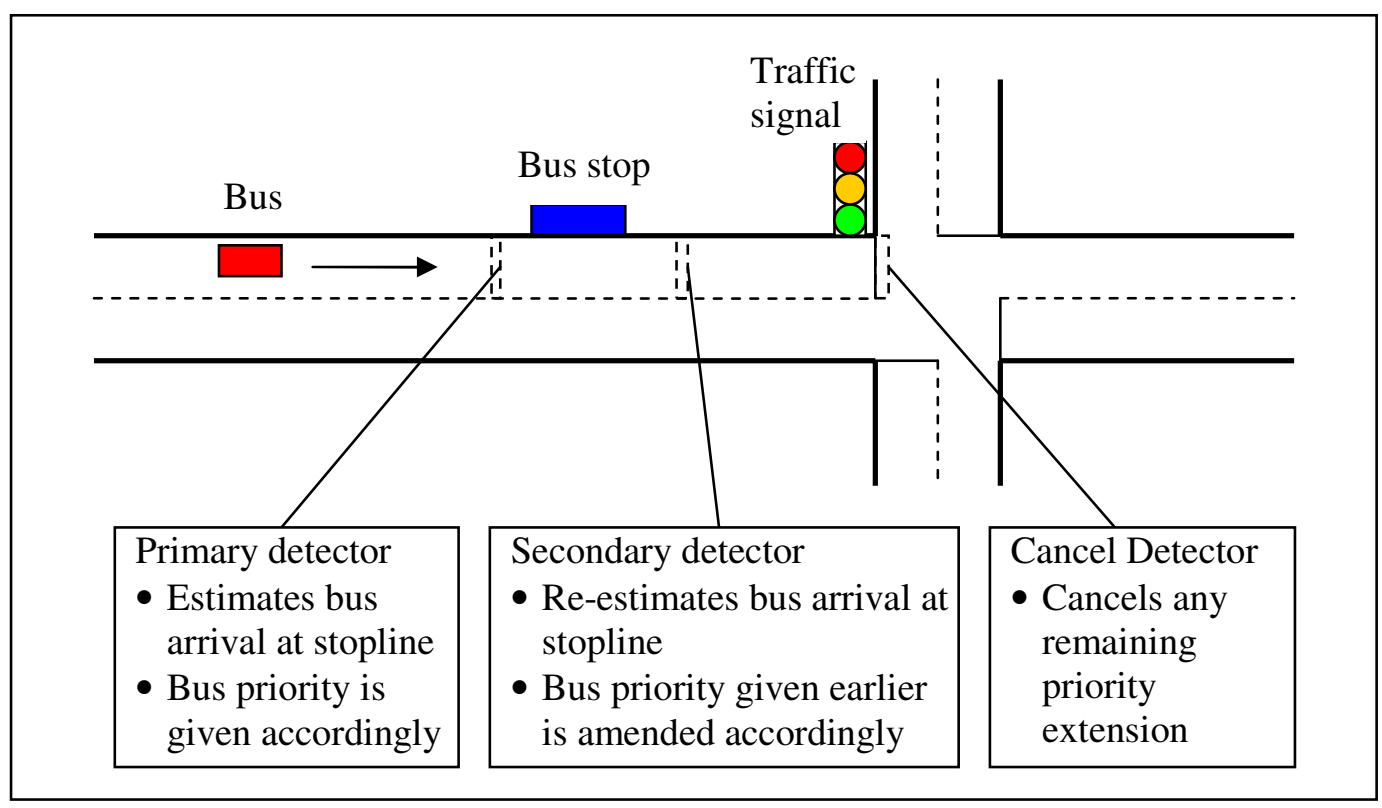

Figure 4: A typical layout of detectors at various locations

\subsection{Differential priority}

Differential priority is the term used where buses receive different levels of priority at traffic signals according to their adherence to schedule/frequency. Differential priority allows different types of priority strategies to be implemented so that different categories of buses (in terms of the lateness) can be targeted. For example, no priority to buses on time and priority to late buses. Implementation of such a method requires: the use of an AVL system that continuously provides the location information of buses in a network; and intelligence to calculate the priority requirement of the buses (e.g. in terms of lateness). iBUS has both of these facilities available to implement differential priority at traffic signals.

In iBUS, a bus receives its location every second from its onboard GPS unit. The bus is continuously monitored and polled by the Control Centre at 30-60 second intervals. A bus also sends its arrival time at a bus stop to the Control Centre. Using this information, the control centre updates locations of each bus in the system and calculates headways in relation to the forerunning bus. The calculated headway is compared with the scheduled headway to calculate headway deviation (lateness) of the bus. The headway and the headway deviation calculated are then passed to the bus. The bus sends the deviation to the bus processor (in the traffic signal controller) when sending priority requests (telegrams) at the time of priority trigger. The bus processor receives the deviation in the priority message and uses it to decide the priority level depending on the priority strategy implemented. The priority level is then passed to the signal controller. The traffic signal controller (liaising with the central system) decides the type and amount of priority given to the approaching bus. This depends on the signal status, junction saturation and the priority level assigned. The working of differential priority in iBus is shown in the functional diagram (Figure 5). 


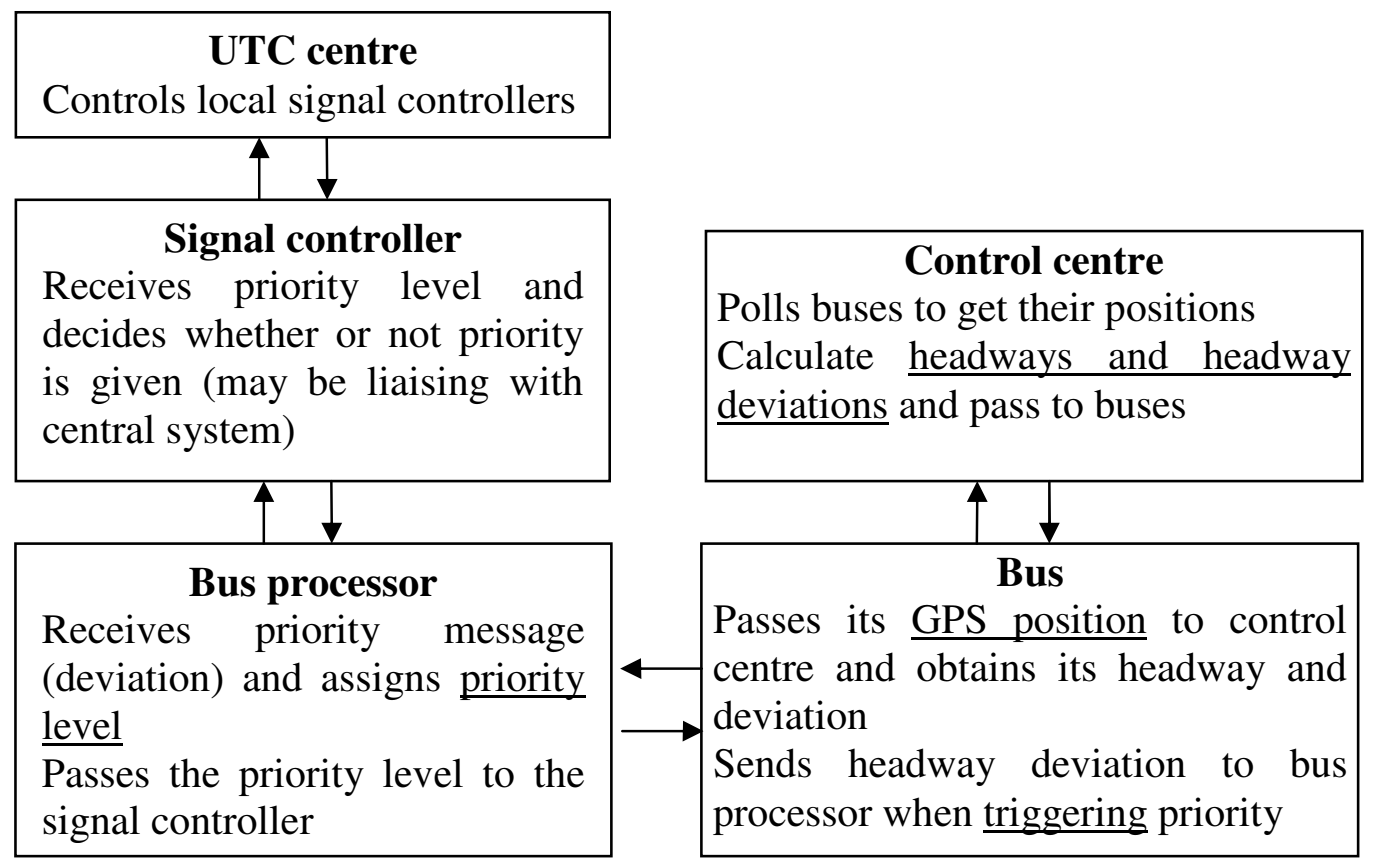

\section{Figure 5: Functional diagram of main components of differential priority in iBUS}

As shown in Figure 5, the main intelligence at the control centre monitors all the buses in a network to calculate the headway deviation (lateness). This overall view of a network could be utilised in future to improve priority decision making taking account of bus operations on the route/network as a whole rather than just at each individual junction. For example, the reference headway could be changed to reflect the bus operation situation in the network for a time period. The usual way of calculating the 'lateness' of buses in headway-based high frequency operations is that the present headway is compared against the scheduled headway. However in some cases, it may not be possible to achieve the scheduled headway by all buses due to operational (e.g. fewer buses available due to a bus breakdown) and network conditions (e.g. congestion). In such cases, the priority algorithm targeting scheduled headway may not be appropriate as nearly all buses may get priority when most headways are higher than scheduled. In this situation, regularity would not be greatly improved. However, if such network information is available and can be processed, it could then be beneficial to use the average headway of buses in the field rather than the scheduled headway. iBUS has such information available which could be used for this purpose.

\subsection{System monitoring}

Experience with existing systems shows that continuous performance monitoring is essential to ensure that the system is working as expected and the original level of benefits are maintained. iBUS provides a comprehensive set of automatic monitoring facilities to analyse equipment and configuration failures. For bus priority at traffic signals, iBUS monitoring is focussed on the critical area where interactions occur between buses and signals [2]. The performance of individual junctions and individual buses (as they pass along the route) can be analysed in detail. The system can also be configured to report automatically when a bus journey time through a link increases above a pre-determined threshold. Similarly, the system can be configured to report automatically when the performance of a radio link between buses and signals deteriorates. Furthermore, the system could be integrated with the existing traffic monitoring system available to enhance performance. The information available from 
such a system could be used to evaluate the impact of bus priority in terms of emissions (particularly green house gases such as $\mathrm{CO}_{2}$ ). Research is currently being carried out at the University of Southampton to look into this aspect of advanced bus priority at traffic signals. This automatic monitoring facility available in iBus should save considerable time and resources required in earlier systems to collect and analyse data.

\section{Conclusions}

London already has a very successful bus priority system at traffic signals. To keep up to the pace of development of new technologies and the possibilities for improving benefits and reliability it opens, TfL has recently procured a GPS-based AVL system known as iBUS. Despite various opportunities it provides, the system also introduces challenges related to the potential bus locational accuracy which could reduce benefits. The challenge posed by the GPS locational error was studied using theoretical analysis as well as simulation modelling. The study of the effect of the GPS error showed that this is more or less constant over all the detector positions considered. This is due to the combined effect of both more green extensions and higher existing bus journey time variability occurring at greater detection distances (balancing each other to give a more or less constant effect). Assuming reported levels of accuracy for GPS location are achieved in London, the effects of GPS locational errors should only reduce bus delay savings by $2-5 \%$ compared to the equivalent bus detection using loops or beacons.

The opportunities for using extra detectors offered by GPS detection for bus priority have also been discussed. These can be exploited to improve bus priority performance. One such example is the use of multiple virtual detectors to monitor buses upstream so that priority can better match the actual movement of a bus from further upstream as it approaches each traffic signal. In addition, iBUS having the information of a whole network at one place can be used to implement the differential bus priority that takes account of the current network situation. In the context of this new implementation, some of the opportunities available and the way ahead presented in the paper will be useful in building even more efficient and beneficial bus priority at traffic signals in London.

\section{Acknowledgements}

The authors would like to acknowledge that earlier version of this paper was presented at $14^{\text {th }}$ ITS World Congress in Beijing which was then substantially enhanced to publish in this journal.

\section{References}

1 Hounsell N.B. and McLeod F.N.: 'Automatic Vehicle Location and Bus Priority: The London System', in Selected Proceedings of the 8th World Conference on Transport Research, 1999, Volume 2, pp. 279-292

2 Bowen, T., Head, J. R., Hounsell, N. B., Palmer, S. and Shrestha, B. P.: 'Expanding the Boundaries of Bus Priority at Traffic Signals in London' in Proceedings of 6th European Congress on Intelligent Transport Systems and services, Aalborg, 2007

3 PRISCILLA: 'Public Transport Priority: State of the Art Review'. Bus Priority Strategies and Impact Scenarios Development on a Large Urban Area - Deliverable 2, February 2002

4 Hounsell, N.B., Shrestha, B.P.: 'AVL based Bus priority architectures: A review of London example’, Eur. J. Transp. Infrastruct. Res., 2005, 5, (3), pp. 13-29 
$5 \quad$ Hounsell N.B., Shrestha B.P., McLeod F.N., Palmer S., Bowen T. and Head J.R.. Using Global Positioning System for Bus Priority in London: Traffic Signals Close to Bus Stops. IET Intelligent Transport Systems, 2007, 1, (2), pp.131-137.

6 Ibrahim, D.: 'Improving Accuracy for GPS Vehicle Navigation Systems in London', Traffic Eng. Control, 2000, 41, (6), pp. 228-232

7 Hounsell, N.B., Ishtiaq, S. and McLeod, F.N.: Journey Time Prediction for Bus Priority at Traffic Signals, Proceedings of the 24th PTRC European Transport Forum: Transportation Management and Safety, London, 1996, vol. 416.

8 Shrestha, B.P.: 'Simulating Advanced Bus Priority Strategies at Traffic Signals', PhD Thesis, University of Southampton, 2003

9 Hounsell, N.B., McLeod, F.N. and Shrestha, B.P.: 'Bus Priority at Traffic Signals: Investigating the Options'. Proceedings of 12th International Conference on Road Traffic Information and Control, IEE London, April 2004

10 Shrestha, B.P., Hounsell, N.B. and McLeod, F.N.: 'Simulating Advanced Bus Priority Strategies at Traffic Signals'. Proceedings of 10th World Conference on Transport Research, Istanbul, July 2004

11 Gillam, W.J., Wright, D.A.: 'An Innovative Approach to Real-Time Bus Information and Signal Priority'. Proceedings of 10th International Conference on Road Traffic Information and Control, IEE London, 2000 